\title{
Tiliacora triandra, an Anti-Intoxication Plant, Improves Memory Impairment, Neurodegeneration, Cholinergic Function, and Oxidative Stress in Hippocampus of Ethanol Dependence Rats
}

\author{
Nattaporn Phunchago, ${ }^{1,2}$ Jintanaporn Wattanathorn, ${ }^{2,3}$ and Kowit Chaisiwamongkol ${ }^{2,4}$ \\ ${ }^{1}$ Department of Physiology (Neuroscience Program) and Graduate School, Faculty of Medicine, Khon Kaen University, \\ Khon Kaen 40002, Thailand \\ ${ }^{2}$ Integrative Complementary Alternative Medicine Research and Development Center, Khon Kaen University, \\ Khon Kaen 40002, Thailand \\ ${ }^{3}$ Department of Physiology, Faculty of Medicine, Khon Kaen University, Khon Kaen 40002, Thailand \\ ${ }^{4}$ Department of Anatomy, Faculty of Medicine, Khon Kaen University, Khon Kaen 40002, Thailand
}

Correspondence should be addressed to Jintanaporn Wattanathorn; jintanapornw@yahoo.com

Received 24 September 2014; Revised 8 February 2015; Accepted 1 March 2015

Academic Editor: Cristina Angeloni

Copyright (C) 2015 Nattaporn Phunchago et al. This is an open access article distributed under the Creative Commons Attribution License, which permits unrestricted use, distribution, and reproduction in any medium, provided the original work is properly cited.

\begin{abstract}
Oxidative stress plays an important role in brain dysfunctions induced by alcohol. Since less therapeutic agent against cognitive deficit and brain damage induced by chronic alcohol consumption is less available, we aimed to assess the effect of Tiliacora triandra extract, a plant possessing antioxidant activity, on memory impairment, neuron density, cholinergic function, and oxidative stress in hippocampus of alcoholic rats. Male Wistar rats were induced ethanol dependence condition by semivoluntary intake of alcohol for 15 weeks. Alcoholic rats were orally given T. triandra at doses of 100, 200, and $400 \mathrm{mg} \cdot \mathrm{kg}^{-1} \mathrm{BW}$ for 14 days. Memory assessment was performed every 7 days while neuron density, activities of AChE, SOD, CAT, and GSH-Px and, MDA level in hippocampus were assessed at the end of study. Interestingly, the extract mitigated the increased escape latency, AChE and MDA level. The extract also mitigated the decreased retention time, SOD, CAT, and GSH-Px activities, and neurons density in hippocampus induced by alcohol. These data suggested that the extract improved memory deficit in alcoholic rats partly via the decreased oxidative stress and the suppression of AChE. Therefore, T. triandra is the potential reagent for treating brain dysfunction induced by alcohol. However, further researches are necessary to understand the detail mechanism and possible active ingredient.
\end{abstract}

\section{Introduction}

Alcohol (ethanol) consumption in Thailand is dramatically increased. Data obtained from alcohol consumption collected by World Health Organization have demonstrated that Thailand is the top ethanol-consuming country in Asia and 40\% of drinkers are in North-East region [1]. Chronic ethanol exposure can produce multiple and durable changes in the central nervous system. It has been reported that chronic ethanol consumption produces a significant loss of brain tissues especially in forebrain and hippocampus [2] together with the neurodegeneration of cholinergic neurons in basal forebrain [3]. Several lines of evidence from animal study have also revealed that a specific neuronal loss in the dentate gyrus, increased arborizations of the dendritic spines of the granule cells, a reduction of the number of spines of the CA3-pyramidal cells, and a reorganization of synaptic formations [4-7] are also presented. In addition, chronic ethanol consumption also induces memory impairment. This impairment has been demonstrated to relate with hippocampus degeneration and cholinergic function [8].

Accumulative lines of evidence have demonstrated that neurodegeneration induced by chronic ethanol consumption is associated with the elevation of oxidative stress $[9,10]$. 
The elevation of oxidative stress induced by chronic ethanol consumption is reported to occur both via the increased free radical formation and via the decreased antioxidant enzyme activities [11]. In addition, these changes can be mitigated by the substances possessing antioxidant activity [10]. Currently, drugs which target at protecting against brain damage and memory impairment in alcoholism are less available. Therefore, the therapeutic benefit of herbal medicine has gained much attention especially in Asian countries [12].

Tiliacora triandra (Colebr.) Diels or Ya-nang in Thai belongs to the family of Menispermaceae. It is the native plant of Southeast Asia and widely used in the cuisines of northeast Thailand and Laos. Ya-nang is used not only as food but also as medicine in traditional folklore. According to the traditional medicine of many countries in Southeast Asia, it has been used as anti-pyretic, detoxication agent, anti-inflammation, anticancer, antibacterial, and immune modulator. In addition, it also possesses antioxidant activity [13]. Recent toxicity study has revealed that water extract of $T$. triandra leaves shows no toxicity up to $5000 \mathrm{mg} \cdot \mathrm{kg}^{-1}$ in single administration. Moreover, no adverse effects were observed following the subchronic administration of the water extract of this plant at doses of 300,600 , and $1200 \mathrm{mg} \cdot \mathrm{kg}^{-1}$ [14]. Based on the antioxidant effect together with the detoxification reputation of this herb and the benefit of substance possessing antioxidant activity against ethanol neurotoxicity mentioned earlier, the health benefit against neurotoxicity of T. triandra extract has been considered. Thus, this study was carried out to determine the effect of water extract of T. triandra on memory impairment, neurodegeneration, cholinergic function, and oxidative stress in hippocampus of ethanol dependence rats.

\section{Materials and Methods}

2.1. Experimental Animals. Adult male Wistar rats, 8 weeks old, were used as experimental animals. They were purchased from National Laboratory Animal Center, Salaya, Nakorn Pathom. All animals were acclimatized for two weeks on normal diet of rat chow, with water given ad libitum at room temperature with a 12-hour light and dark cycle before the commencement of the experiment. The weights of the animals on the first day of experiment were 180$220 \mathrm{~g}$. The experiments were performed to minimize animals' suffering and the experiment protocols were approved by the Institutional Animal Care and Unit Committee Khon Kaen University, Thailand.

2.2. Plant Material and Extract Preparation. The aerial parts of $T$. triandra were collected from Khon Kaen province, Thailand. The plant was authenticated by Associate Professor Panee Siri-sa-ard, Faculty of Pharmacy, Chiangmai University, Chiangmai, Thailand (authentication number 023160). They were cleaned dried and ground to fine powder. Then, the ground powder was boiled with distilled water at a ratio of $1: 6(\mathrm{w} / \mathrm{v})$ for 5 minutes with a continuous stirring. After being left at room temperature for 24 hours, the extract was filtered using Whatman No. 1. The filtrate was evaporated under reduced pressure using a rotary evaporator [15]. The percent yield of the extract was 5.5.
TABLE 1: Gradient program of HPLC analysis.

\begin{tabular}{lcc}
\hline \multirow{2}{*}{ Times (minutes) } & \multicolumn{2}{c}{ Solvents (\%) } \\
& A (methanol) & B (2.5\% acetic acid) \\
\hline 0 & 10 & 90 \\
17 & 70 & 30 \\
18 & 100 & - \\
20 & 100 & - \\
20.5 & 10 & 90 \\
25 & 10 & 90 \\
\hline
\end{tabular}

2.3. Sample Analysis. The total phenolic compounds content was determined with the Folin-Ciocalteu reagent. Gallic acid was used as a standard and the total phenolics were expressed as $\mathrm{mg} / \mathrm{g}$ gallic acid equivalents (GAE) [16].

The finger print of $T$. triandra leaves extract was carried out by using gradient high performance liquid chromatography (HPLC) system. The system consists of 515 HPLC pump and 2998 Photodiode array detector of Waters company, USA. Chromatographic separation was performed using Purospher STAR, C-18 encapped ( $5 \mu \mathrm{m})$, LiChroCART 2504.6, and HPLC-Cartridge, Sorbet Lot number HX255346 (Merk, Germany). Two mobile phases consisting of methanol and $2.5 \%$ acetic acid in deionized (DI) water were used to induce gradient elution. The injection volume was $20 \mu \mathrm{L}$ and the flow rate was $1.0 \mathrm{~mL} / \mathrm{min}$. During HPLC analysis the solvent gradient was programmed as shown in Table 1 and data analysis was performed using Empower 3.

2.4. Experimental Protocol. The animals were induced ethanol dependence by using a semivoluntary intermittent intake method [17]. In brief, rats were exposed to ethanol in drinking water. The ethanol concentration in drinking water was gradually increased in a stepwise fashion from 5\% at a rate of $5 \%$ per week until reaching a $20 \%$ ethanol wihin 4 weeks in order to allow the animals to get used to the taste of alcohol and mimic the condition of alcohol addiction in human. Then, the ethanol concentration was raised to $30 \%$ ethanol and maintained at this concentration between the fifth and the fifteenth weeks. The hyperexcitability symptoms which reflected ethanol dependence condition including the increased response to environmental stimuli such as startle, irritability, and hypervigilance were evaluated [17]. In addition, all ethanol dependence rats used in this study showed the average ethanol concentration at $9.89 \pm 0.86 \mathrm{mg} / \mathrm{dL}$ prior to the intervention. The ethanol dependence rats were selected for further study and randomly assigned to 7 groups of 6 animals each as follows.

Group I: control group: rats in this group received no treatment.

Group II: ethanol + vehicle: alcoholic rats were orally administered vehicle (distilled water).

Group III: ethanol + Aricept (donepezil): ethanol dependence rats were administered donepezil via oral route at dose of $1 \mathrm{mg} / \mathrm{kg}^{-1} \mathrm{BW}$ (this group served as positive control because this drug has been used as 
standard drug for treating memory deficit patients) [18].

Group IV: ethanol + vitamin C: ethanol dependence rats were administered vitamin $\mathrm{C}$ at dose of $250 \mathrm{mg} / \mathrm{kg}^{-1} \mathrm{BW}$. (This group also served as positive control based on the previous findings that the substances possessing antioxidant effect exert neuroprotective effect against alcohol neurotoxicity of vitamin C [19].)

Group V: ethanol + T. triandra 100: ethanol dependence rats were orally administered $T$. triandra extract at dose of $100 \mathrm{mg} \cdot \mathrm{kg}^{-1} \mathrm{BW}$.

Group VI: ethanol + T. triandra 200: ethanol dependence rats were orally administered $T$. triandra extract at dose of $200 \mathrm{mg} \cdot \mathrm{kg}^{-1} \mathrm{BW}$,

Group VII: ethanol + T. triandra 400: ethanol dependence rats were orally administered $T$. triandra extract at dose of $400 \mathrm{mg} \cdot \mathrm{kg}^{-1} \mathrm{BW}$.

All animals received the assigned treatments at a period of 14 days after the induction of ethanol dependence by a 15week alcohol treatment. They were assessed spatial memory using Morris water maze test after the single intervention, 7 days and 14 days of treatments. At the end of experiment, they were sacrificed and hippocampi were isolated for the determination of oxidative damage markers including the level of malondialdehyde (MDA) and the activities of catalase (CAT), superoxide dismutase (SOD), and glutathione peroxidase (GSH-Px). In addition, the activity of acetylcholinesterase (AChE) in hippocampus, the crucial area for learning and memory, was also determined.

2.5. Determination of Spatial Memory. Spatial memory was evaluated using Morris water maze test. According to this test, a circular pool $(160 \mathrm{~cm}$ in diameter $\times 60 \mathrm{~cm}$ height $)$ filled with water (approximately $42 \mathrm{~cm}$ deep, temperature of 23 $24^{\circ} \mathrm{C}$ ) was divided into 4 quadrants. The removable platform was placed in the center on one quadrant under the water surface. The immersed platform was invisible due to the masking effect of nontoxic milk powder which covered the water surface. The animal must memorize the location of the immersed platform by forming the association memory between its location and the location of the platform via the environmental cues. The time which the animal spent to find the hidden platform and climb onto the platform was recorded as escape latency. The platform was removed $24 \mathrm{hr}$ later and the animal was reexposed to the test again to evaluate the memory retention and retrieval capacity. The time which the animals spent swimming in the quadrant which the platform was previously located was recorded as the retention time $[20,21]$.

2.6. Determination of Oxidative Stress Markers. Rats were perfused with cold saline solution to get rid of the blood from the brain tissue; then, hippocampi were rapidly removed and stored at $-80^{\circ} \mathrm{C}$ until used. To determine the oxidative stress markers, brains were prepared as homogenate and we determined the level of malondialdehyde (MDA) using the thiobarbituric acid reaction [22] whereas glutathione peroxidase (GSH-Px), catalase (CAT), and superoxide dismutase (SOD) were determined using a spectrophotometric method [23].

2.6.1. Determination of Malondialdehyde (MDA) Level. Level of malondialdehyde (MDA), a relatively stable lipid peroxidation marker, was determined by using thiobarbituric acid reacting substances (TBARS) assay. MDA which occurred as the result of the breakdown of polyunsaturated fatty acid must react with thiobarbituric acid reacting substances and gives rise to the pink color product. This product could be measured at $532 \mathrm{~nm}$. In this test, 1,3,3-tetra ethoxy propane (TEP) was used as the standard.

2.6.2. Catalase (CAT) Assay. Catalase activity was determined spectrophotometrically by measuring the decrease in $\mathrm{H}_{2} \mathrm{O}_{2}$ absorbance at $490 \mathrm{~nm}$. A system devoid of the substrate (hydrogen peroxide) served as the control. The difference in absorbance per unit time was expressed as the activity. One unit was defined as the amount of enzyme required to decompose $1.0 \mathrm{M}$ of hydrogen peroxide per minute at $\mathrm{pH} 7.0$ and $25^{\circ} \mathrm{C}$.

2.6.3. Superoxide Dismutase (SOD) Assay. The activity of SOD was performed using a xanthine/xanthine oxidase system for the production of superoxide radical and subsequent measurement of cytochrome $\mathrm{c}$ as a scavenger of the radicals. Optical density was determined using a spectrometer (UV1601, Shimadzu) at $550 \mathrm{~nm}$. A system devoid of enzyme served as control. One unit of enzyme activity was defined as the quantity of SOD required to inhibit the rate of reduction of cytochrome by $50 \%$. SOD activity was presented as units per milligram of protein (U/mg protein).

2.6.4. Glutathione Peroxidase (GSH-Px) Assay. The determination of GSH-Px was performed using t-butyl hydroperoxide as a substrate. The optical density was recorded at $340 \mathrm{~nm}$. One unit of the enzyme was defined as micromole $(\mu \mathrm{mol})$ of reduced nicotinamide adenine dinucleotide phosphate (NADPH) oxidized per minute. GSH-Px activity was expressed as $\mathrm{U} / \mathrm{mg}$ protein.

2.7. Determination of Acetylcholinesterase (AChE) Activity. Acetylcholinesterase (AChE) was analyzed based on the basis that this enzyme catalyzed the hydrolysis of acetylthiocholine (ATCh) which in turn gave rise to the formation of acetate and thiocholine. In the presence of the highly reactive 5,5'-dithiobis-(2-nitrobenzoic acid) (DTNB) ion, thiocholine generated a yellow color substance which was quantitatively monitored by spectrophotometric absorption at $405 \mathrm{~nm}$ [24].

\subsection{Histological Study}

2.8.1. Tissue Preparation. At the end of study, all rats were exposed to transcardial perfusion. In brief, the thorax was carefully cut open, the heart was exposed, and a needle 
connected to the tubing from the fixative bottle was inserted into the left ventricle. The right atrium was cut open to drain out the blood and fixative. First $20-30 \mathrm{~mL}$ of saline was passed transcardially to flush out the blood and then perfuse with $4 \%$ paraformaldehyde in $0.1 \mathrm{M}$ phosphate buffer $\mathrm{pH} 7.4$. Fixation was monitored by the gradual discolorations of the tongue and eyeball. After fixation has been established, the brain specimens were further kept in the fixative containing $30 \%$ sucrose for $72 \mathrm{~h}$. Regions of hippocampus were then dissected out using Paxinos stereotaxic coordinate method [25]. Serial sections of tissues containing hippocampus area were prepared using a sliding microtome at $20 \mu \mathrm{m}$ thick. Tissue samples were picked up on slides coated with a $0.01 \%$ aqueous solution poly-L-lysine.

2.8.2. Cresyl Violet Staining of Brain Tissue. The sections containing hippocampus of all groups were stained with $0.5 \%$ cresyl violet. Analysis of neuronal density was performed on coronal sections of the dorsal hippocampus stained with cresyl violet and corresponding to brain sections located between 3.14 and $4.16 \mathrm{~mm}$ posterior to bregma [25]. The densities of living neurons in CA1, CA2, CA3, and dentate gyrus were performed using Olympus light microscope model $\mathrm{BH}-2$ at $40 \mathrm{x}$ magnification by an observer who was blind to the treatment at time of analysis.

2.9. Statistical Analysis. Data were presented as mean \pm standard error of mean (SEM). Statistical analysis was analyzed using one-way analysis of variance (ANOVA), followed by LSD post hoc test. Probability levels less than 0.05 were accepted as significance.

\section{Results}

3.1. Sample Analysis. The data obtained from this study showed that the T. triandra leaves extract used in this study contained total phenolic compounds at concentration of $593.33 \mathrm{mg}$ of gallic acid equivalent (GAE)/mg extract. The HPLC fingerprint of $T$. triandra was shown in Figure 1 . We had identified gallic acid, cyanidin, and quercetin according to their retention times and spectral characteristics of their peaks compared with standard. The ultraviolet spectrum of chromatographic bands presented in the fingerprinting of the samples indicated the presence of gallic acid at a concentration of $4.81 \pm 0.05 \mu \mathrm{g}$ gallic/100 $\mathrm{mg}$ of extract whereas the concentrations of cyanidins and quercetin were presented at the concentrations of 307.22 $\pm 4.74 \mu \mathrm{g}$ Cyn-3-glu/100 mg and $9028.86 \pm 695.97 \mu \mathrm{g}$ QE/100 mg extract, respectively.

\subsection{Effect of T. triandra Extract on Spatial Memory. Figure 2} showed that ethanol dependence rats induced by 15 -week alcohol consumption which received vehicle or water showed the enhanced escape latency since the first day of treatment until the end of 14-day study period $(P$ value $<0.001$ all, compared to control rats). Donepezil, vitamin C, and all doses of T. triandra failed to modulate the enhanced escape latency induced by ethanol at 1-day treatment period. However, when the treatments were prolonged to 7 and 14 days, ethanol

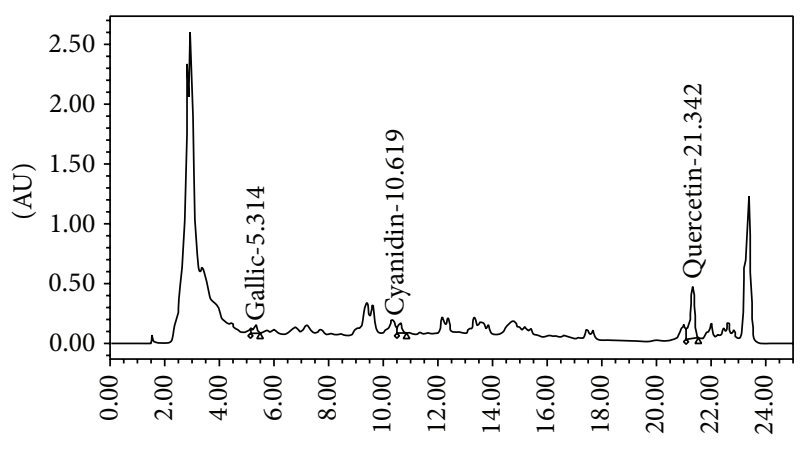

(min)

FIgURE 1: The chromatogram of water extract of T. triandra.

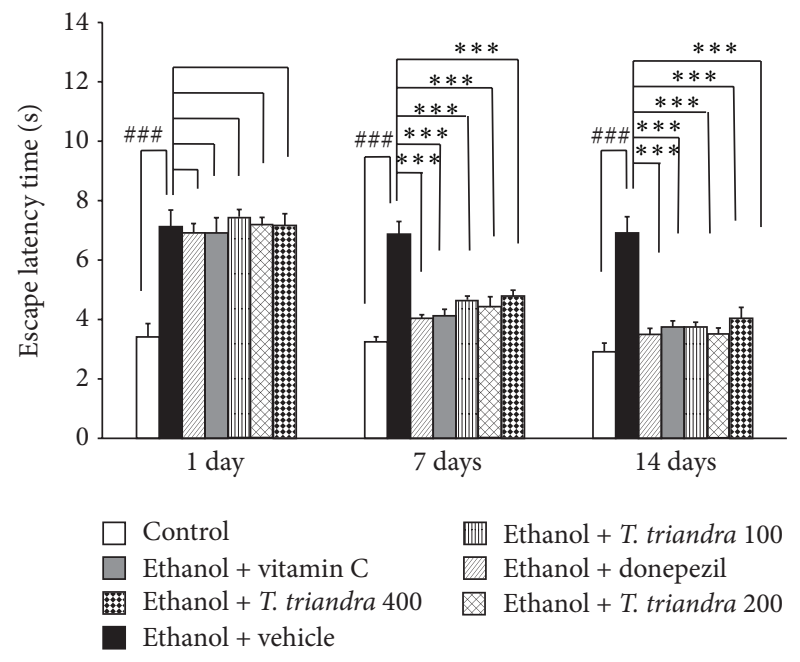

FIgure 2: The effect of T. triandra on escape latency time in Morris water maze test. Data were presented as mean \pm SEM, $n=$ $6 /$ group. ${ }^{\# \# \#} P$ value $<0.001$ compared with control treated group. ${ }^{* * * *} P$ value $<0.001$ compared with ethanol dependence treated group which received vehicle.

dependence rats which received donepezil or vitamin $\mathrm{C}$ or T. triandra extract at doses of 100,200 , and $400 \mathrm{mg} \cdot \mathrm{kg}^{-1} \mathrm{BW}$ significantly mitigated the elevation of escape latency induced by ethanol $(P$ value $<0.001$ all, compared with ethanol dependence rats which received vehicle).

The effect of $T$. triandra on retention time in Morris water maze test was also investigated and results were shown in Figure 3. It was found that ethanol dependence rats which received vehicle showed the decreased retention time throughout the 14 -day study period $(P$ value $<0.001$ all, compared to control rats). Ethanol dependence rats which received either donepezil or vitamin $\mathrm{C}$ significantly mitigated the decreased retention time induced by alcohol consumption at 7 and 14 days of treatment $(P$ value $<0.001$ all, compared with ethanol dependence rats which received vehicle). In addition, ethanol dependence rats which received T. triandra at doses of 200 and $400 \mathrm{mg} \cdot \mathrm{kg}^{-1} \mathrm{BW}$ also showed the significant attenuation of the decreased retention time induced by ethanol consumption both at $7(P$ value $<0.001$ and 0.05 , resp., compared with ethanol dependence rats 


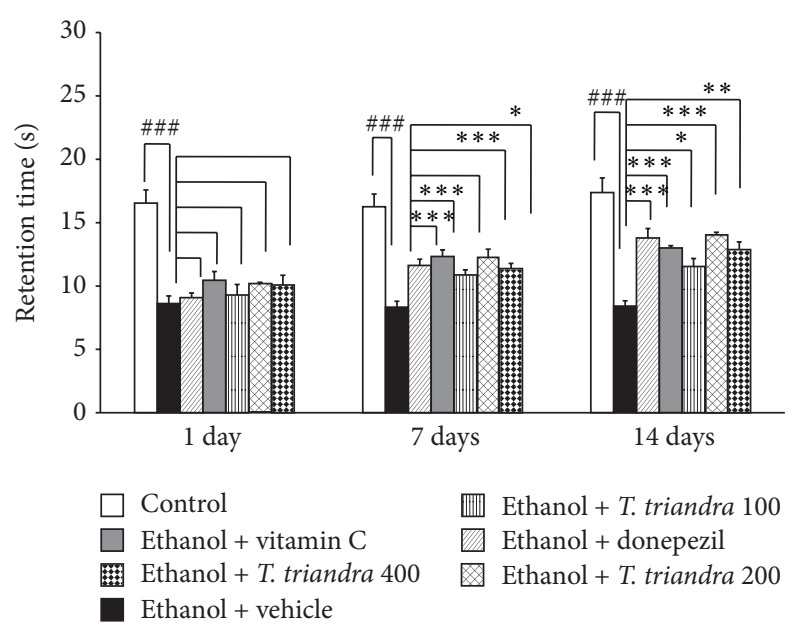

FIgURE 3: The effect of T. triandra on retention time in Morris water maze test. Data were presented as mean \pm SEM, $n=6 /$ group. ${ }^{\# \# ~} P$ value $<0.001$ compared with control treated group. ${ }^{*} P$ value $<0.05$ compared with ethanol dependence treated group which received vehicle. ${ }^{* * *} P$ value $<0.001$ compared with ethanol dependence treated group which received vehicle.

which received vehicle) and 14 days of treatments ( $P$ value $<0.001$ all, compared with ethanol dependence rats which received vehicle). However, ethanol dependence rats which received T. triandra at dose of $100 \mathrm{mg} \cdot \mathrm{kg}^{-1} \mathrm{BW}$ significantly attenuated the decreased retention time induced by ethanol consumption only at 14 days of treatment $(P$ value $<0.05$, compared with ethanol dependence rats which received vehicle).

\subsection{Effect of T. triandra on AChE Activity in Hippocampus.} Since cholinergic system played an important role on spatial memory, we also investigated the effect of $T$. triandra on AChE activity in this area. The results were shown in Figure 4. The significant elevation of AChE activity in hippocampus was observed in ethanol dependence rats which received vehicle ( $P$ value $<0.001$, compared with control rats). Treatment with either donepezil or vitamin $C$ could mitigate the elevation of AChE in hippocampus ( $P$ value $<0.01$ all, compared with ethanol dependence rats which received vehicle). Ethanol dependence rats which received the extract at dose of $200 \mathrm{mg} \cdot \mathrm{kg}^{-1} \mathrm{BW}$ also significantly mitigated an elevation of AChE activity induced by ethanol consumption in hippocampus $(P$ value $<0.01$ all, compared with ethanol dependence rats which received vehicle) while no changes were observed in ethanol dependence rats which received low and high doses of extract.

\subsection{Effect of T. triandra on Neuron Density in Hippocampus.} Effect of T. triandra on neuron density in various subregions of hippocampus including CA1, CA2, CA3, and dentate gyrus was shown in Figures 5(a) and 5(b). Repetitive consumption of alcohol significantly decreased neuron density in CA1, CA2, CA3, and dentate gyrus of hippocampus $(P$ value $<$ 0.001 all, compared with control rats). Ethanol dependence

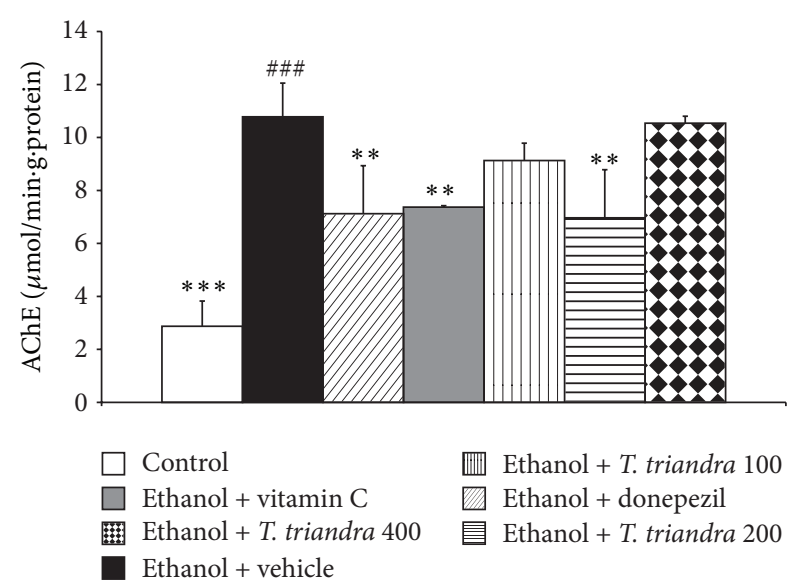

FIgURE 4: The effect of T. triandra on the activity of acetylcholinesterase (AChE) in hippocampus. Data were presented as mean \pm SEM, $n=6 /$ group ${ }^{\# \# \# ~} P$ value $<0.001$ compared with control treated group. ${ }^{* *} P$ value $<0.01$ compared with ethanol dependence treated group which received vehicle. ${ }^{* * *} P$ value $<0.001$ compared with ethanol dependence treated group which received vehicle.

rats which received either donepezil or vitamin $\mathrm{C}$ significantly attenuated the decreased neuron density induced by alcohol in all subregions mentioned earlier of hippocampus $(P$ value $<0.01$ all, compared with ethanol dependence rats which received vehicle). Interestingly, all doses of $T$. triandra extract treatment significantly counteracted the decreased neuron density induced by alcohol consumption in hippocampus $(P$ value $<0.01$ all, compared with ethanol dependence rats which received vehicle).

3.5. Effect of T. triandra on Oxidative Stress Markers. Table 2 demonstrated the effects of T. triandra on oxidative stress markers including malondialdehyde (MDA) level and the activities of main scavenger enzymes such as superoxide dismutase (SOD), catalase (CAT), and glutathione peroxidase (GSH-Px) in hippocampus. The current data showed that alcohol consumption significantly enhanced MDA level $(P$ value $<0.001$, compared with control rats) but decreased SOD, CAT, and GSH-Px ( $P$ value $<0.001,0.01$, and 0.01 , resp., compared with control rats) enzyme activities. Treatments with donepezil, vitamin C, and T. triandra at doses of 100, 200 , and $400 \mathrm{mg} \cdot \mathrm{kg}^{-1} \mathrm{BW}$ produced the significant mitigation effect on the elevation of MDA level $(P$ value $<0.01$ all, compared with alcoholic rats which received vehicle) in hippocampus of ethanol dependence rats. The significant attenuation of SOD activity induced by ethanol in hippocampus was also observed in ethanol dependence rats which received donepezil or vitamin $C$ or T. triandra at doses of 100,200 , and $400 \mathrm{mg} \cdot \mathrm{kg}^{-1} \mathrm{BW}(P$ value $<0.001,0.001,0.01$, 0.001 , and 0.01 , resp., compared with ethanol dependence rats which received vehicle). In addition, ethanol dependence rats treated with donepezil showed a significant attenuation effect on the decreased CAT and GSH-Px activities induced by alcohol $(P$ value $<0.01$, all, compared with ethanol dependence rats which received vehicle) whereas those which received 


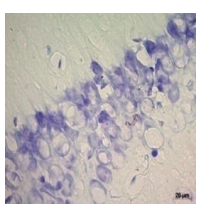

Control

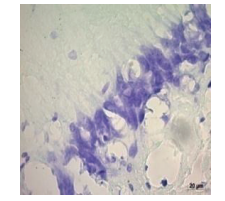

Ethanol + Ethanol
vehicle

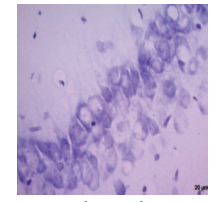

Ethanol +

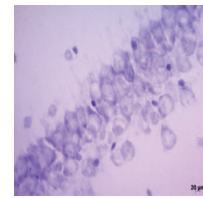

Ethanol +

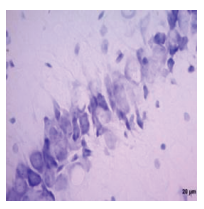

Ethanol + T. triandra 100

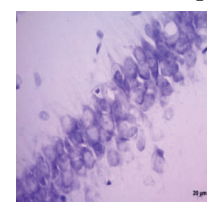

Ethanol + T. triandra 200

(1)

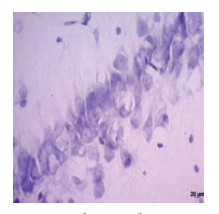

Ethanol + T. triandra 400

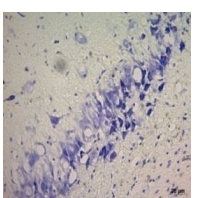

Control

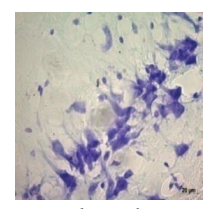

Ethanol + vehicle

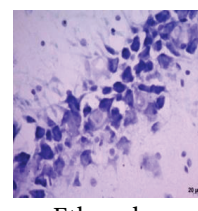

Ethanol + donepezil

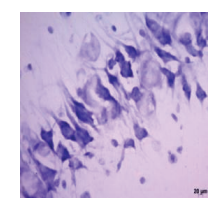
Ethanol +
vitamin C

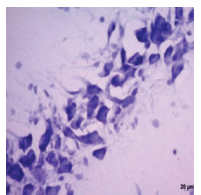

Ethanol + T. triandra 100

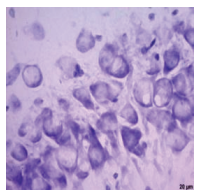

Ethanol + T. triandra 200

(3)

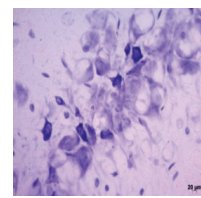

Ethanol + T. triandra 400

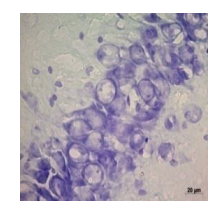

Control

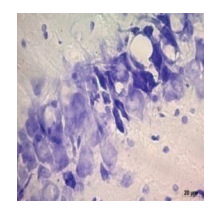

Ethanol +

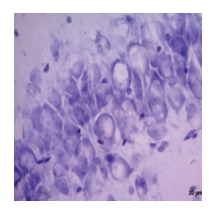

Ethanol +

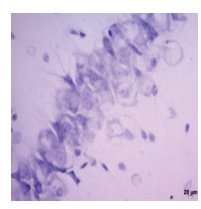

Ethanol + vitamin C

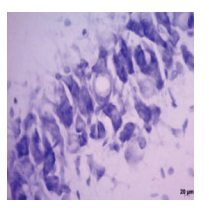

Ethanol + T. triandra 100

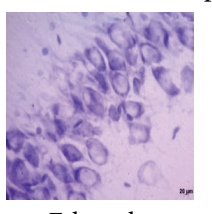

Ethanol + T. triandra 200

(2)

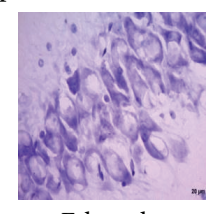

Ethanol +

T. triandra 400

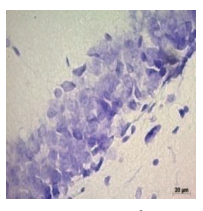

Control

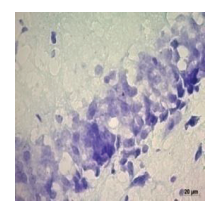

Ethanol + vehicle

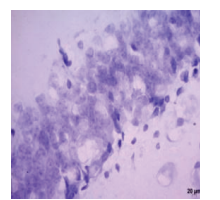

Ethanol + donepezil

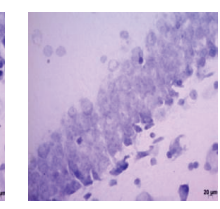

Ethanol +
vitamin C

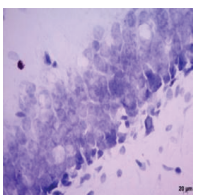

Ethanol +

T. triandra 100

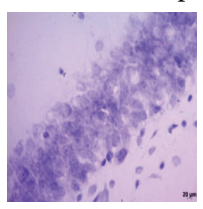

Ethanol +

T. triandra 200

(4)

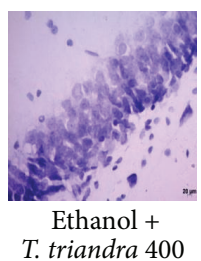

T. triandra 400

(a)

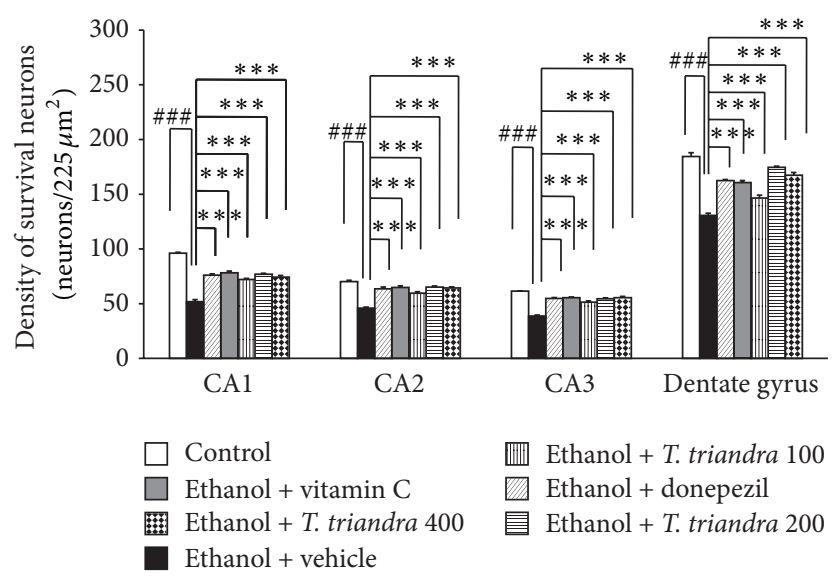

(b)

FIgure 5: The effect of T. triandra extract on the neuron density in CA1, CA2, CA3, and dentate gyrus of hippocampus. (a) Photographs showing the density of the neurons stained with cresyl violet; (b) the bar graph showing density of neurons in various subregions of hippocampus. Data were presented as mean \pm SEM, $n=6$ /group. ${ }^{\# \# *} P$ value $<0.001$ compared with control treated group. ${ }^{* * *} P$ value $<$ 0.001 compared with ethanol dependence treated group which received vehicle.

vitamin $C$ showed only the significant change of CAT activity in hippocampus $(P$ value $<0.05$, compared with ethanol dependence rats which received vehicle). It was found that T. triandra extract at doses of 100,200 , and $400 \mathrm{mg} \cdot \mathrm{kg}^{-1} \mathrm{BW}$ significantly mitigated the enhanced MDA level induced by ethanol consumption $(P$ value $<0.001$ all, compared with ethanol dependence rats which received vehicle). The significant increased SOD activity in hippocampus was also observed in alcoholic rats which were treated with extract at all doses used in this study $(P$ value $<0.01,0.001$, and 0.01 , resp., compared with ethanol dependence rats which received vehicle). However, the significant increased CAT activity in 
TABLE 2: The effect of T. triandra extract on oxidative stress markers including malondialdehyde (MDA) level and the activities of superoxide dismutase (SOD), catalase (CAT), and glutathione peroxidase (GSH-Px) in hippocampus.

\begin{tabular}{lcccc}
\hline Groups/oxidative stress markers & $\begin{array}{c}\text { MDA } \\
\text { (nmol/mg·protein) }\end{array}$ & $\begin{array}{c}\text { SOD } \\
(\mathrm{U} / \mathrm{mg} \cdot \text { protein) }\end{array}$ & $\begin{array}{c}\text { CAT } \\
(\mathrm{U} / \mathrm{mg} \cdot \text { protein) }\end{array}$ & $\begin{array}{c}\text { GSH-Px } \\
(\mathrm{U} / \mathrm{mg} \cdot \mathrm{protein})\end{array}$ \\
\hline Control & $0.0017 \pm 0.0002^{* * *}$ & $2.80 \pm 0.39^{* * *}$ & $12.38 \pm 0.61^{* *}$ & $3.473 \pm 0.43^{* * *}$ \\
Ethanol + vehicle & $0.012 \pm 0.0007^{\# \# \#}$ & $0.98 \pm 0.31^{\# \# \#}$ & $8.65 \pm 1.73^{\# \#}$ & $2.473 \pm 0.18^{\# \#}$ \\
Ethanol + donepezil & $0.005 \pm 0.0003^{* * *}$ & $2.80 \pm 0.39^{* * *}$ & $13.18 \pm 1.95^{* *}$ & $4.620 \pm 0.61^{* *}$ \\
Ethanol + vitamin C & $0.004 \pm 0.0007^{* * *}$ & $2.80 \pm 0.39^{* * *}$ & $10.91 \pm 0.64^{*}$ & $3.013 \pm 0.10$ \\
Ethanol + T. triandra 100 & $0.005 \pm 0.0005^{* * *}$ & $1.62 \pm 0.43^{* *}$ & $12.877 \pm 0.72^{* *}$ & $4.30 \pm 0.53^{* *}$ \\
Ethanol + T. triandra 200 & $0.004 \pm 0.0005^{* * *}$ & $2.12 \pm 0.27^{* * *}$ & $11.55 \pm 1.09^{* *}$ & $6.29 \pm 0.52^{* * *}$ \\
Ethanol + T. triandra 400 & $0.004 \pm 0.0004^{* * *}$ & $1.56 \pm 0.18^{* *}$ & $9.31 \pm 1.63$ & $3.92 \pm 0.56^{* *}$ \\
\hline
\end{tabular}

Values are expressed as means \pm SEM from 6 animals in each group.

${ }^{\# \#} P$ value $<0.01$ and ${ }^{\# \#} P$ value $<0.001$ compared with control treated group.

${ }^{*} P$ value $<0.05,{ }^{* *} P$ value $<0.01$, and ${ }^{* * *} P$ value $<0.001$ compared with ethanol dependence rats which received vehicle.

hippocampus was observed only in ethanol dependence rats which were treated with low and medium doses of extract $(P$ value $<0.01$ all, compared with ethanol dependence rats which received vehicle). Treatment with $T$. triandra at the dosage range used in this study also mitigated the decrease of GSH-Px activity in hippocampus of ethanol dependence rats ( $P$ value $<0.01,0.001$, and 0.01 , resp., compared with ethanol dependence rats which received vehicle).

\section{Discussion}

The data obtained from this study demonstrated that the ethanol dependence rats used in this study were hyperactive and increased responsiveness to environmental stimuli, such as startle, increased puffing and hair ruffling, irritability, and hypervigilance similar to that observed in the previous study [17]. The average ethanol concentration in the blood of all ethanol dependence rats prior to the intervention was $9.89 \pm$ $0.86 \mathrm{mg} / \mathrm{dL}$. According to this model, it was found that no serious signs of physical dependence such as spasticity and convulsion were observed. Therefore, the animal model used in this study successfully induced mild to moderate ethanol dependence.

It was found that ethanol dependence rats induced by repetitive consumption of alcohol showed the increased escape latency but decreased retention time together with the neurodegeneration of hippocampus and hypocholinergic function. This was in agreement with the previous studies $[4-8,26,27]$. It has been reported that chronic alcohol consumption induces hippocampal damage, spatial memory impairment [28], together with the decreased oxidative stress [19]. Therefore, the results obtained from this study suggested that the elevation of oxidative stress reflected by the increased MDA level in hippocampus was responsible for the hippocampal damage and memory impairment. In addition, the decreased cholinergic function induced by chronic ethanol consumption also plays the important role in the improved hippocampal damage and memory impairment. It was found that donepezil and vitamin $\mathrm{C}$ could attenuate an enhanced oxidative stress and a cholinergic impairment which in turn improved memory deficit $[10,29,30]$. In addition, T. triandra leaves extract at all doses used in this study could also mitigate both the enhanced escape latency and the decreased retention time. Moreover, it also increased neuron density and improved oxidative stress status and cholinergic function in hippocampus of ethanol dependence rats. Previous studies clearly demonstrated that the neurons in hippocampus play the crucial role in the spatial memory [31]. Therefore, we did suggest that the improved oxidative stress which in turn increased neuron density in hippocampus was responsible for the improved memory impairment in ethanol dependence rats which received T. triandra leaves extract. In addition, the improved cholinergic function also played a role in the improved memory deficit in ethanol dependence rats which received a medium dose of $T$. triandra leaves extract. It was found that high dose of the extract failed to show the suppression of AChE in hippocampus. The possible explanation might be associated with the masking effect of the other ingredients in the crude extract of $T$. triandra leaves. Since T. triandra contained high concentration of polyphenolic compounds and high concentration of quercetin and these compounds also exerted neuroprotective effect against various insults [32-36], we did suggest that the neuroprotective effect of $T$. triandra extract observed in this study might be partly associated with quercetin. However, this still required further investigation.

\section{Conclusions}

The current study clearly demonstrates that Tiliacora triandra provides neuroprotective and cognitive enhancing effects in ethanol dependence rats. Therefore, it should be used as functional food and nutraceutical product to improve memory impairment and brain damage induced by ethanol dependence. The possible mechanism of extract may be due to the antioxidant and the suppression of acetylcholinesterase activity in hippocampus. Further studies are required to investigate the possible active ingredients, detail mechanism, and signal pathways.

\section{Conflict of Interests}

The authors declare that there is no conflict of interests regarding the publication of this paper. 


\section{Acknowledgment}

This study was supported by the National Research Council of Thailand, Invitation Research of Faculty of Medicine and Integrative Complementary Alternative Medicine Research and Development Center, Khon Kaen University, Khon Kaen, Thailand.

\section{References}

[1] World Health Organization, "Country profile: South-East Asia region," in WHO Global Status Report on Alcohol 2004, pp. 1-5, World Health Organization, Geneva, Switzerland, 2004.

[2] I. Agartz, R. Momenan, R. R. Rawlings, M. J. Kerich, and D. W. Hommer, "Hippocampal volume in patients with alcohol dependence," Archives of General Psychiatry, vol. 56, no. 4, pp. 356-363, 1999.

[3] T. Arendt, "Impairment in memory function and neurodegenerative changes in the cholinergic basal forebrain system induced by chronic intake of ethanol," Journal of Neural Transmission, Supplement, no. 44, pp. 173-187, 1994.

[4] M. M. Paula-Barbosa, F. Brandao, M. D. Madeira, and A. Cadete-Leite, "Structural changes in the hippocampal formation after long-term alcohol consumption and withdrawal in the rat," Addiction, vol. 88, no. 2, pp. 237-247, 1993.

[5] A. Cadete-Leite, M. A. Tavares, and M. M. Paula-Barbosa, "Alcohol withdrawal does not impede hippocampal granule cell progressive loss in chronic alcohol-fed rats," Neuroscience Letters, vol. 86, no. 1, pp. 45-50, 1988.

[6] A. Cadete-Leite, M. A. Tavares, M. M. Pacheco, B. Volk, and M. M. Paula-Barbosa, "Hippocampal mossy fiber-CA3 synapses after chronic alcohol consumption and withdrawal," Alcohol, vol. 6, no. 4, pp. 303-310, 1989.

[7] N. V. Lukoyanov, F. Brandão, A. Cadete-Leite, M. D. Madeira, and M. M. Paula-Barbosa, "Synaptic reorganization in the hippocampal formation of alcohol-fed rats may compensate for functional deficits related to neuronal loss," Alcohol, vol. 20, no. 2, pp. 139-148, 2000.

[8] F. Melis, R. Stancampiano, A. Imperato, G. Carta, and F. Fadda, "Chronic ethanol consumption in rats: correlation between memory performance and hippocampal acetylcholine release in vivo," Neuroscience, vol. 74, no. 1, pp. 155-159, 1996.

[9] J. Haorah, S. H. Ramirez, N. Floreani, S. Gorantla, B. Morsey, and Y. Persidsky, "Mechanism of alcohol-induced oxidative stress and neuronal injury," Free Radical Biology \& Medicine, vol. 45, no. 11, pp. 1542-1550, 2008.

[10] J. Wattanathorn, N. Phunchago, S. Muchimapura et al., "Mulberry fruit mitigates alcohol neurotoxicity and memory impairment induced by chronic alcohol intake," American Journal of Applied Sciences, vol. 9, no. 4, pp. 484-491, 2012.

[11] M. A. Collins and E. J. Neafsey, "Ethanol and adult CNS neurodamage: oxidative stress, but possibly not excitotoxicity," Frontiers in Bioscience, vol. 4, no. 4, pp. 1358-1367, 2012.

[12] E. Ernst, "Prevalence of use of complementary/alternative medicine: a systematic review," Bulletin of the World Health Organization, vol. 78, no. 2, pp. 252-257, 2000.

[13] S. Rattana, M. Padungkit, and B. Cushnie, "Phytochemical screening, flavonoid content, and antioxidant activity of Tiliacora triandra leaf extracts," in Proceedings of the 2nd Annual International Conference of Northeast Pharmacy Research, pp. 60-63, February 2010.
[14] S. Sireeratawong, N. Lertprasertsuke, U. Srisawat et al., "Acute and subchronic toxicity study of the water extract from Tiliacora triandra (Colebr.) Diels in rats," Songklanakarin Journal of Science and Technology, vol. 30, no. 5, pp. 611-619, 2008.

[15] M. M. Al-Dabbas, K. Al-Ismail, K. Kitahara et al., "The effects of different inorganic salts, buffer systems, and desalting of Varthemia crude water extract on DPPH radical scavenging activity," Food Chemistry, vol. 104, no. 2, pp. 734-739, 2007.

[16] Y. Y. Lim, T. T. Lim, and J. Jing, "Antioxidant properties of guava fruit: comparison with some local fruits," Sunway Academic Journal, vol. 3, pp. 9-20, 2006.

[17] M. S. Macieira, W. G. Almeida, E. A. Silva, L. C. Schenberg, and E. M. Nakamura-Palacios, "Alcohol dependence induced in rats by semivoluntary intermittent intake," Brazilian Journal of Medical and Biological Research, vol. 30, no. 9, pp. 1107-1111, 1997.

[18] M. Cochrane, A. Cochrane, P. Jauhar, and E. Ashton, "Acetylcholinesterase inhibitors for the treatment of WernickeKorsakoff syndrome-three further cases show response to donepezil," Alcohol and Alcoholism, vol. 40, no. 2, pp. 151-154, 2005.

[19] E. Macit, G. Ulusoy, T. Celik, H. Kayir, and T. Uzbay, “Comparative effects of antioxidants on chronic ethanol-induced oxidative stress in rat hippocampus," Journal of Neurological Sciences, vol. 29, no. 2, pp. 329-339, 2012.

[20] M. L. Byrnes, D. P. Richardson, J. F. Brien, J. N. Reynolds, and H. C. Dringenberg, "Spatial acquisition in the Morris water maze and hippocampal long-term potentiation in the adult guinea pig following brain growth spurt-prenatal ethanol exposure," Neurotoxicology and Teratology, vol. 26, no. 4, pp. 543-551, 2004.

[21] R. Morris, "Developments of a water-maze procedure for studying spatial learning in the rat," Journal of Neuroscience Methods, vol. 11, no. 1, pp. 47-60, 1984.

[22] H. Ohkawa, N. Ohishi, and K. Yagi, "Assay for lipid peroxides in animal tissues by thiobarbituric acid reaction," Analytical Biochemistry, vol. 95, no. 2, pp. 351-358, 1979.

[23] C. J. Weydert and J. J. Cullen, "Measurement of superoxide dismutase, catalase and glutathione peroxidase in cultured cells and tissue," Nature Protocols, vol. 5, no. 1, pp. 51-66, 2010.

[24] G. L. Ellman, K. D. Courtney, V. Andres Jr., and R. M. Featherstone, "A new and rapid colorimetric determination of acetylcholinesterase activity," Biochemical Pharmacology, vol. 7, no. 2, pp. 88-95, 1961.

[25] G. Paxinos and C. Waston, The Rats Brain in Stereotaxic Coordinates, Academic Press, San Diego, Calif, USA, 4th edition, 1998.

[26] R. Khalil, M. A. King, and M. R. I. Soliman, "Testosterone reverses ethanol-induced deficit in spatial reference memory in castrated rats," Pharmacology, vol. 75, no. 2, pp. 87-92, 2005.

[27] V. Tiwari and K. Chopra, "Protective effect of curcumin against chronic alcohol-induced cognitive deficits and neuroinflammation in the adult rat brain," Neuroscience, vol. 244, pp. 147-158, 2013.

[28] A. M. White, P. E. Simson, and P. J. Best, "Comparison between the effects of ethanol and diazepam on spatial working memory in the rat," Psychopharmacology, vol. 133, no. 3, pp. 256-261, 1997.

[29] K. Nixon, "Alcohol and adult neurogenesis: roles in Neurodegeneration and recovery in chronic alcoholism," Hippocampus, vol. 16, no. 3, pp. 287-295, 2006.

[30] M. Jamal, K. Ameno, S. Ameno et al., "Changes in cholinergic function in the frontal cortex and hippocampus of rat exposed to ethanol and acetaldehyde," Neuroscience, vol. 144, no. 1, pp. 232-238, 2007. 
[31] M.-B. Moser and E. I. Moser, "Distributed encoding and retrieval of spatial memory in the hippocampus," The Journal of Neuroscience, vol. 18, no. 18, pp. 7535-7542, 1998.

[32] K. B. Pandey and S. I. Rizvi, "Plant polyphenols as dietary antioxidants in human health and disease," Oxidative Medicine and Cellular Longevity, vol. 2, no. 5, pp. 270-278, 2009.

[33] S. Schaffer and B. Halliwell, "Do polyphenols enter the brain and does it matter? Some theoretical and practical considerations," Genes and Nutrition, vol. 7, no. 2, pp. 99-109, 2012.

[34] N. Sriraksa, J. Wattanathorn, S. Muchimapura, S. Tiamkao, K. Brown, and K. Chaisiwamongkol, "Cognitive-enhancing effect of quercetin in a rat model of Parkinson's disease induced by 6-hydroxydopamine," Evidence-Based Complementary and Alternative Medicine, vol. 2012, Article ID 823206, 9 pages, 2012.

[35] K. Selvakumar, S. Bavithra, G. Krishnamoorthy, P. Venkataraman, and J. Arunakaran, "Polychlorinated biphenyls-induced oxidative stress on rat hippocampus: a neuroprotective role of quercetin," The Scientific World Journal, vol. 2012, Article ID 980314, 10 pages, 2012.

[36] N. A. Kelsey, H. M. Wilkins, and D. A. Linseman, "Nutraceutical antioxidants as novel neuroprotective agents," Molecules, vol. 15, no. 11, pp. 7792-7814, 2010. 


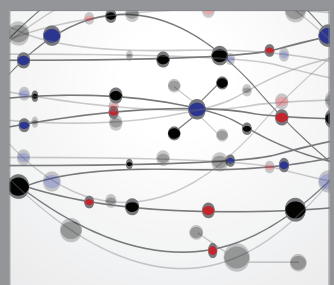

The Scientific World Journal
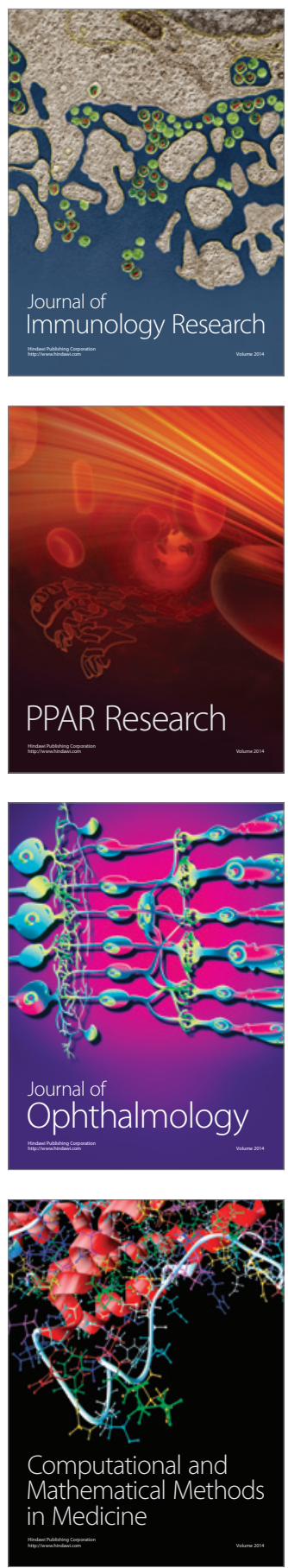

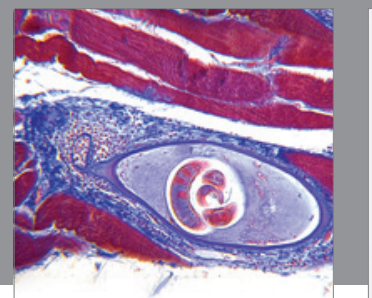

Gastroenterology

Research and Practice
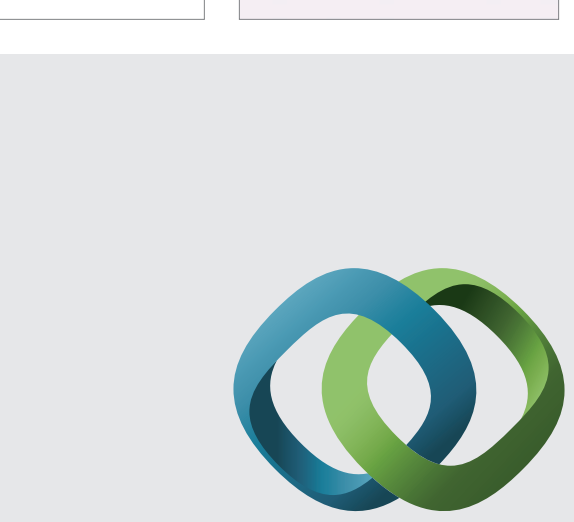

\section{Hindawi}

Submit your manuscripts at

http://www.hindawi.com
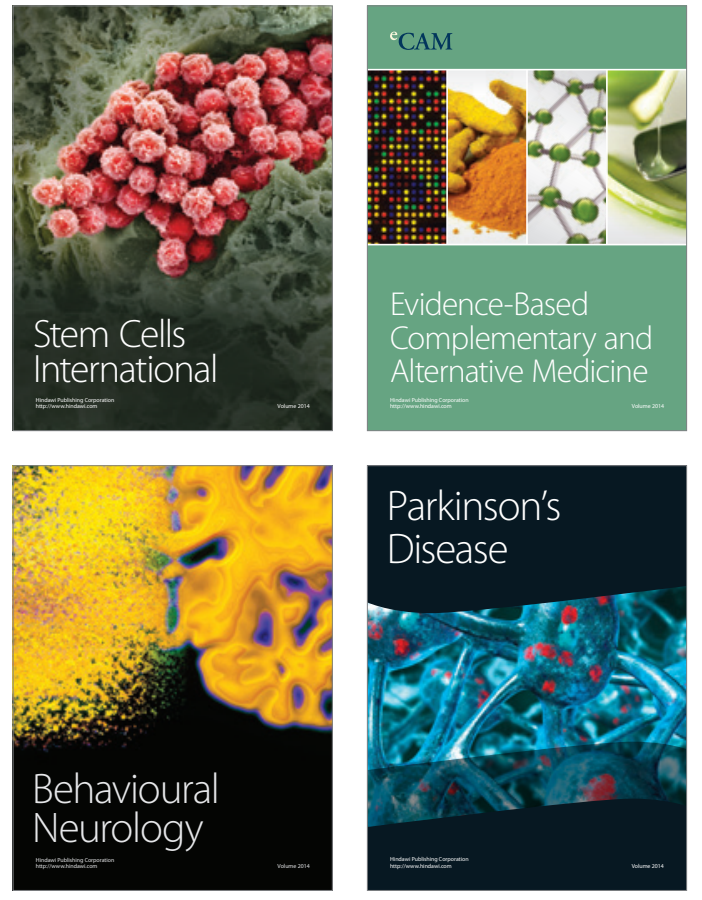
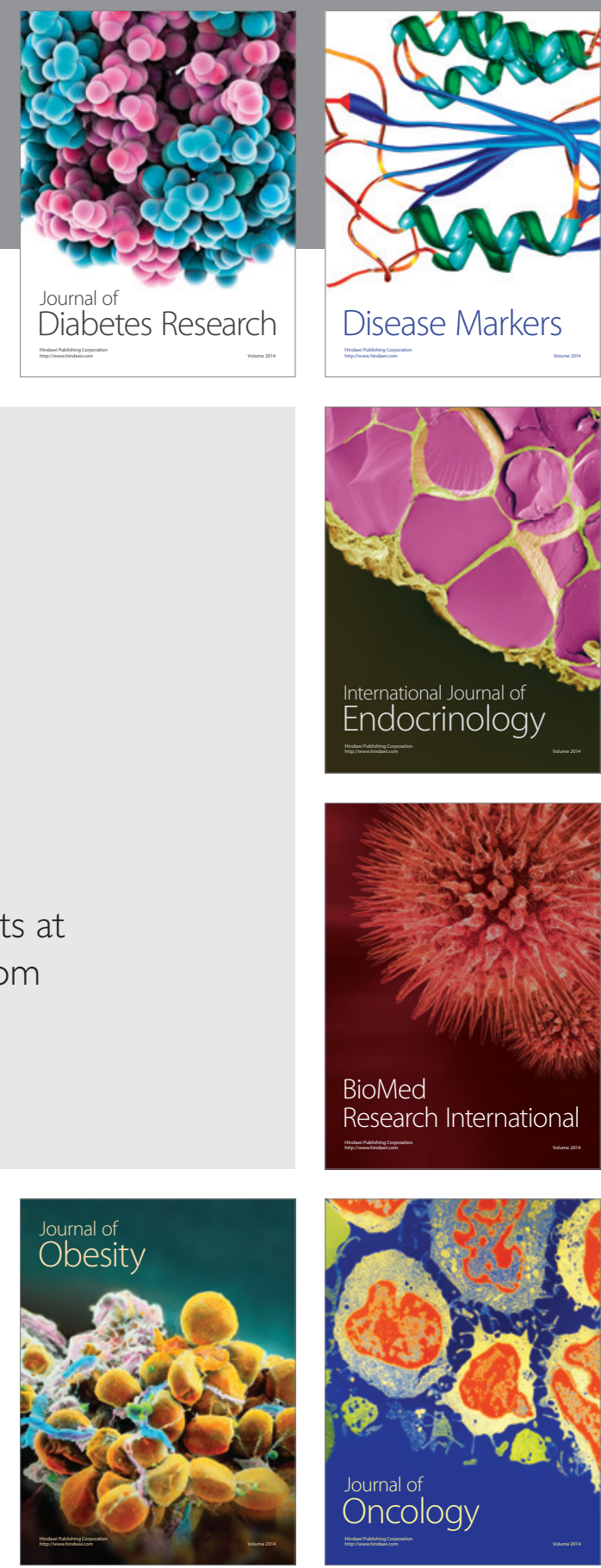

Disease Markers
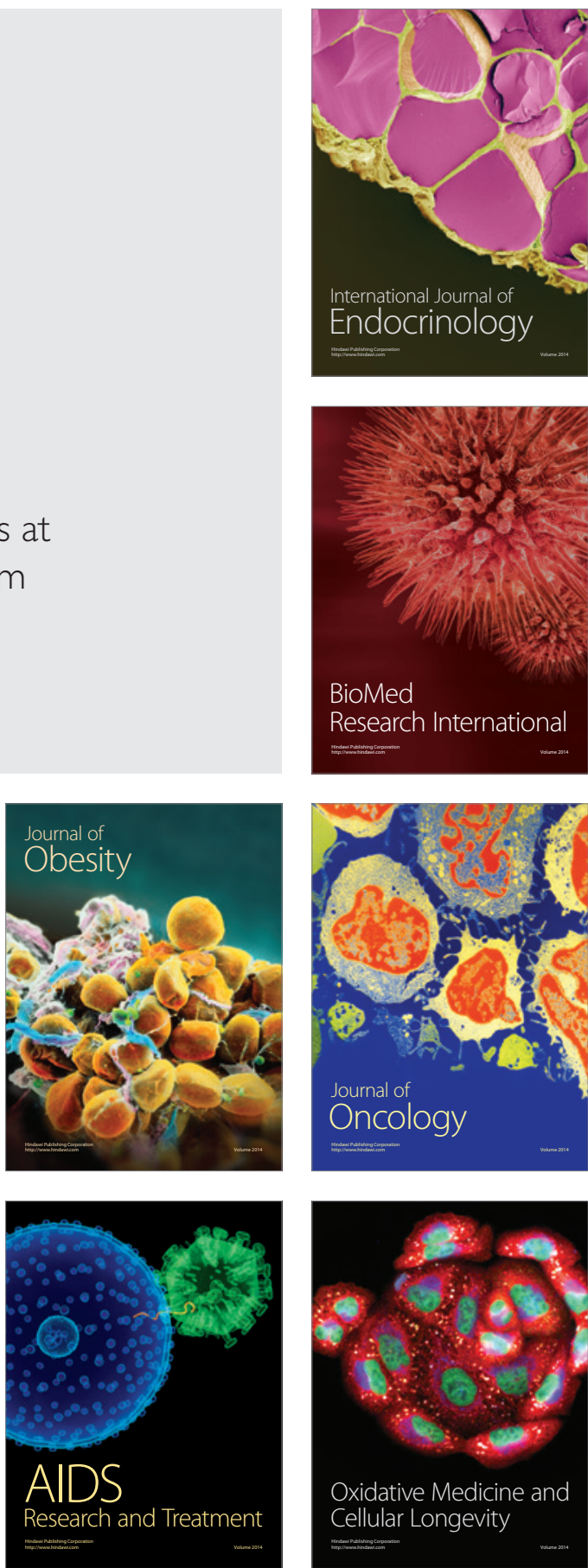